patients. 3. After a follow-up time of 3.5 years, $43 \%$ of patients that died had been diagnosed as a IPAF.

References:

[1] Fischer A,et al. Er Respir J 2015.

[2] Raghu G, et al.Am J Respir Crit Care Med 2011.

Disclosure of Interest: None declared

DOI: 10.1136/annrheumdis-2017-eular.5550

\section{AB1058 SENSITIVE DETECTION OF DYNAMIC CHANGES OF BONE EROSIONS IN INFLAMMATORY ARTHRITIS BY MUSCULOSKELETAL ULTRASOUND: A COMPARATIVE ANALYSIS WITH HIGH-RESOLUTION PERIPHERAL QUANTITATVE COMPUTED TOMOGRAPHY}

S. Finzel ${ }^{1,2}$, G. Schett ${ }^{2}$, S. Kraus ${ }^{3}$, R. Voll ${ }^{1}$, M. Backhaus ${ }^{4} .{ }^{1}$ Department of Rheumatology and Clinical Immunology, University Medical Center Freiburg, Freiburg; ${ }^{2}$ Department of Internal Medicine III, Rheumatology and Immunology, University Clinic of Erlangen, Erlangen, Germany; ${ }^{3}$ Department of Internal Medicine, Kontonsspital Baden, Baden, Switzerland: ${ }^{4}$ Department of Internal Medicine, Rheumatology and Clinical Immunology, Park-Klinik Weissensee, Academic Hospital of Charité, Berlin, Germany

Background: Bone erosion is a hallmark of inflammatory joint diseases. Its meticulous detection is highly important for correct diagnosis and monitoring of therapy response. Earlier studies showed that musculoskeletal ultrasound (MSUS) has a higher sensitivity than conventional radiography with regards to detection of bone erosions (1) making MSUS more and more popular. The OMERACT ultrasound working group is currently standardizing and validating MSUS as an imaging outcome tool.

Objectives: To investigate the ability of MSUS to sensitively and specifically detect bone erosions in a longitudinal setting using HR-pQCT as a gold standard. Methods: This is a sequel study to our 2011 cross-sectional comparative analysis on MSUS and HR-pQCT (2). 4/6 healthy individuals, $6 / 6$ psoriatic arthritis patients and of 10/14 rheumatoid arthritis patients were available for follow-up and received an MSUS and an HR-pQCT scan of the clinically dominant hand. Bone erosions at the radial, palmar, and dorsal sites of the second metacarpophalangeal (MCP) joint, as well as the palmar and dorsal sites of the third and forth MCP joints were assessed for prevalence and severity in MSUS and by HR-pQCT. Afterwards, data were compared to the 2011-dataset. MSUS was graded as described earlier (2)

Results: Datasets without follow-up from the baseline cohort were eliminated. Sensitivity of MSUS in comparison to HR-pQCT regarding correct detection of erosions was $95 \%$ and specificity was $75 \%$. \%. For this analysis, grade 1 lesions were included. At follow-up sensitivity was $86 \%$ and specificity $79 \%$. At follow-up, 36 MSUS-lesions were no longer detectable in MSUS; 21/36 were false-positive lesions at baseline. Only one false-positive lesion was detected at both time points. One new lesion was detected by MSUS and confirmed by HR-pQCT. Overall severity of bone erosions regressed in MSUS; these findings were confirmed by HR-pQCT $(p=0.04)$.

Conclusions: This is the first study on change of bone erosions over time comparing MSUS and HR-pQCT. MSUS was confirmed being a sensitive imaging tool able to detect changes of erosions over time. Thus, it may be an adept tool to monitor treatment response in inflammatory joint diseases. Correct identification of bone erosions and differentiation from physiological vessel channels requires knowledge of predilection sites of erosions and physiological cortical breaks; this might aid to further increase the diagnostic value of MSUS

\section{References:}

[1] Wakefield RJ et al. The value of sonography in the detection of bone erosions in patients with rheumatoid arthritis: a comparison with conventional radiography. Arthritis Rheum. 2000.

[2] Finzel $\mathrm{S}$ et al. A detailed comparative study of high-resolution ultrasound and micro-computed tomography for detection of arthritic bone erosions. Arthritis Rheum. 2011.

Disclosure of Interest: None declared

DOI: 10.1136/annrheumdis-2017-eular.6969

\section{AB1059 THE PREVALENCE OF DENSE FINE SPECKLED PATTERN IN ROUTINE SCREENING FOR SYSTEMIC AUTOIMMUNE DISEASES USING INDIRECT IMMUNOFLUORESCENCE-ANTINUCLEAR ANTIBODY TEST}

S. Kim, Y.L. Jeon, M.H. Kim, W.I. Lee, S.Y. Kang. Department of Laboratory Medicine, Kyung Hee University College of Medicine, Seoul, Korea, Republic Of

Background: The nuclear dense fine speckled (DFS) pattern is one of the most commonly observed finding in indirect immunofluorescence-antinuclear antibody (IIF-ANA) assay on HEp-2 cells. Unlike other ANA, DFS pattern is not prevalent in ANA-associated rheumatic diseases (AARD). The antigen was initially named DFS70 (70kD protein) according to the IIF pattern and later known as the lens epithelium-derived growth factor p75 (LEDGF/p75). Autoantibodies showing a DFS pattern have been reported in interstitial cystitis, various chronic inflammatory conditions, autoimmune thyroiditis, atopic dermatitis, cancer, as well as in apparently healthy individuals. Among IIF-ANA tests referred to the laboratory from the Department of Dermatology, the most common IIF-ANA positive pattern is DFS.

Objectives: To compare the clinical significance of DFS pattern in dermatologic diseases (including alopecia) with other departments.

Methods: Between June and December 2016, IIF-ANA testing using HEp-2 cell line slide (Kallestad; Bio-Rad, USA) was performed on 4,130 samples referred as screening for systemic autoimmune diseases in Kyung Hee University Hospital. The identified patterns in IIF-ANA assays were analyzed according clinical department and diagnosis.

Results: The Department of Dermatology was the most requesting IIF-ANA assay $(2579 / 4130,62.4 \%)$ and the Department of Rheumatology and the remaining departments were $18.1 \%(749 / 4130)$ and $19.5 \%$ (802/4130), respectively. The prevalence of IIF-ANA positivity was $10.97 \%(453 / 4130)$ and those of the Department of Dermatology, the Department of Rheumatology, and the remaining departments were $8.5 \%(219 / 2579), 15.5 \%$ (116/749), and $14.7 \%(118 / 802)$, respectively. The DFS pattern was the most common IIF-ANA positive pattern $(173 / 453,38.2 \%)$ and the prevalence of DFS pattern in the Department of Dermatology, the Department of Rheumatology, and the remaining departments were $48.4 \%$ (106/219), $26.7 \%$ (31/116), and 30.5\% (36/118), respectively. Among 173 patients with ANA pattern of DFS, 168 patients were reviewed based on their medical chart. The most of patients were from Department of Dermatology and Rheumatology. 101 patients with positive DFS were from Department of Dermatology, the majority of 55 patients were diagnosed with alopecia. 31 patients of department of Rheumatology showed positive ANA pattern of DFS and a great part of patients were diagnosed with rheumatism.

Conclusions: According to previous studies, up to $20 \%$ of healthy individuals have been reported to have a positive IIF-ANA test and the DFS pattern has been reported in 33\% of ANA positive healthy individuals, but not in ANA positive systemic autoimmune diseases. In this study the prevalence of DFS pattern of ANA positivity in patients with dermatologic diseases including alopecia was similar with prevalence reported in healthy individuals. The patients with alopecia appear to show higher prevalence of positive ANA pattern of DFS than other patients with dermatologic disorders. This study was performed with routinely IIFANA requested patients to screen for systemic autoimmune diseases. Therefore, further evaluations comparing healthy individuals and patient group with more various disease entities are needed to confirm our findings.

Disclosure of Interest: None declared

DOI: 10.1136/annrheumdis-2017-eular.5665

\section{AB1060 MEASURING AGREEMENT IN THE ULTRASONOGRAPHIC EVALUATION OF DISEASE ACTIVITY IN RHEUMATOID ARTHRITIS PATIENTS. A LATIN-AMERICAN MULTICENTER EXERCISE ASSESSING THE INFLUENCE OF SONOGRAPHER EXPERIENCE AND EXPERTISE}

T. Cazenave $^{1}$, M.V. Martire ${ }^{2}$, C. Waimann ${ }^{3}$, M. Rosemffet ${ }^{1} .{ }^{1}$ Instituto de rehabilitación psicofísica, Buenos Aires; ${ }^{2}$ Hospital Italiano de la Plata, la Plata;

${ }^{3}$ Hospital Dr. Hector Cura, Olavarria, Argentina

Background: Ultrasonography (US) is an important tool in rheumatology practice but it depends on sonographer's experience.

Objectives: To evaluate the reliability of US assessment among observers across Latin American using a web tool.

Methods: Cross-sectional study. Fifty-one Latin American ultrasonographers took part in a web- based US exercise evaluating images from 20 RA patients. The 4 joints US score was calculated for each patient including bilateral radiocarpal, midcarpal and second metacarpophalangeal joints. PD and GS were graded from 0 to 3 . US scores comes as the result of the addition of PD and GS score, being 36 the highest disease activity.

Five patients were evaluated twice in order to address intra - rater reliability. The inter and intra-rater reliability was assessed using a two-way random, absolute, individual and average-measures intra-class correlation coefficient (ICC). We stratified sonographers according to experience (defining High experience as: at least 5 years of experience and 80 US assessments/month).

Results: A total of 1020 US image assessments were performed. Mean 4-joints US score was $17 \pm 8$. The ICC was in the excellent range for intra [(individual ICC $=0.945($ IC95\% 0.905-0.965); average ICC =0.972 (IC95\% 0.950-0.982)] and

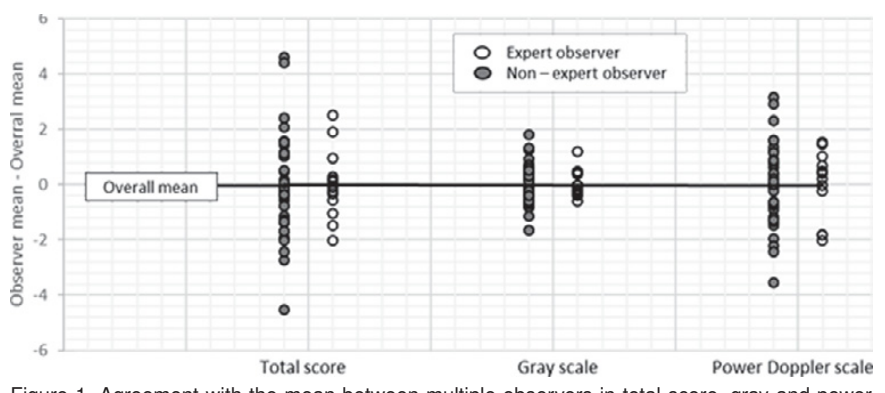

Figure 1, Agreement with the mean between multiple observers in total score, gray and power doppler subscale. 
inter- reader reliability [(individual ICC $=0.867$ (IC95\% 0.786-0.934); average ICC $=0.997($ IC95\% 0.995-0.999)].

When comparing high with low experience sonographers, there was no significant differences in intra-class correlation coefficient. However, there was a greater variation between the means among low experience readers (13 to 22) and higher experience sonographers (14-18) (Figure 1).

Conclusions: US reliability is related to sonographer expertise and experience. Acknowledgements: Edith Alarcón, Lucio Ventura, Walter J. Spindler, Christina Hernandez-Diaz, Javier Rosa, Santiago Ruta, Mara Guinsburg, Gustavo Rodriguez Gil, Cecilia Urquiola, Guillermo Py, Magalí Alva, Patricio Tate, Carmen Cerón, Lida Santiago, Ana Laura Alvarez del Castillo Araujo, Maria Jezabel Haye Salinas, Erika Catay, Maximiliano Bravo, Johana Zacariaz, Clarisa Sandobal, Gonzalo Pacheco, Mariana Benegas, David Navarta, Verónica Arturi, Ana Bertoli, Marcelo Audisio, Carlos Pineda, Natalia Estrella, Carla Airoldi, Paula Kohan, María Julia Santa Cruz, Lina Saldarriaga Rivera, Romulo Wong, Ignacio Carrillo, Hugo Najera, Julio García, Daniele Freitas Pereir, Fernanda Athayde Cardoso Linhares, José Alexandre Mendonça, Maritza Quintero, Anthony M. Reginato, Eliana Natalí Ayala Ledesma, Lorena Urioste, Eugenio De Miquel, María Soledad Gálvez Elkin, Carla Saucedo, Josefina Marin, Rodolfo Arape, Marwin Gutierrez. Disclosure of Interest: None declared

DOI: 10.1136/annrheumdis-2017-eular.5870

\section{AB1061 THE HIGH DOSES GOLIMUMAB BRING BETTER SUPPRESSION OF ULTRASONOGRAPHIC SYNOVIAL INFLAMMATION IN PATIENTS WITH RHEUMATOID ARTHRITIS?}

T. Okano $^{1}$, K. Inui ${ }^{1}$, Y. Sugioka ${ }^{2}$, K. Mamoto $^{1}$, T. Koike ${ }^{2,3}$, H. Nakamura $^{1}$. ${ }^{1}$ Department of Orhopedic surgery; ${ }^{2}$ Center for Senile Degenerative Disorders (CSDD), Osaka City University Graduate School of Medicine, Osaka; ${ }^{3}$ Search Institute for Bone and Arthritis Disease (SINBAD), Shirahama Foundation for Health and Welfare, Wakayama, Japan

Background: Biologic diseasemodifying antirheumatic drugs (bDMARDs) that target cytokines and cytokine receptors such as tumor necrosis factor (TNF)alpha and interleukin (IL)6 have been established as a standard therapy of rheumatoid arthritis (RA) for patients with conventional systemic DMARDs, such as methotrexate (MTX), resistant disease. Golimumab, one of the bDMARDs, is an antibody targeting to TNF-alpha. In Japan, we can choose the dose of golimumab $50 \mathrm{mg}$ or $100 \mathrm{mg}$ according to the disease activity. Recent advance of ultrasound (US) equipment allows obtaining high-quality gray-scale (GS) imaging and sensitive power Doppler (PD) assessment, especially at small joints of the hands and feet. To date, US is the most sensitive imaging modality available in daily rheumatology practice for the assessment of residual synovitis.

Objectives: The aim of this study was to compare the ultrasound findings between patients with rheumatoid arthritis (RA) treated by golimumab $100 \mathrm{mg}$ and 50mg.

Methods: Patients with RA treated by golimumab were consecutively included. Ultrasound examination was performed at 52 synovial sites, bilateral first to fifth MCP, first IP and second to fifth PIP joints, first to fifth flexor tendon and wrists, 2nd and 6th compartment of extensor tendons and first to fifth MTP joints, by using HI VISION Ascendus (Hitachi Medical Corporation, Japan) with a multifrequency linear transducer $(18-6 \mathrm{MHz})$. The GS and PD signals were scored in each synovial site using a semi-quantitative scale from 0 to 3 .

Results: Fifty-five patients with RA (46 female, mean age: $64.2 \pm 12.1$ years) were included and analyzed. In comparison between the dose of Golimumab at the time of ultrasound examination, disease activity (DAS28-CRP) was significantly higher in 100mg group (100mg, $n=15: 3.6 \pm 1.0,50 \mathrm{mg}, n=40: 2.3 \pm 0.9 ; p<0.001$ ), but ultrasound findings were not significantly different between golimumab $100 \mathrm{mg}$ and $50 \mathrm{mg}$ groups. In patients achieving remission, ultrasound findings were not different between $100 \mathrm{mg}$ started and $50 \mathrm{mg}$ started groups.

Table 1. The comparison between golimumab $100 \mathrm{mg}$ and $50 \mathrm{mg}$ RA patients at the time of ultrasound examination

\begin{tabular}{lccc} 
& Golimumab $100 \mathrm{mg}(\mathrm{n}=15)$ Golimumab 50mg $(\mathrm{n}=40)$ & $P$ value \\
\hline Age (years old) & $60.3 \pm 12.2$ & $65.6 \pm 12.0$ & 0.161 \\
Disease duration (years) & $17.5 \pm 9.6$ & $14.2 \pm 9.5$ & 0.263 \\
Duration of golimumab use (years) & $1.8 \pm 0.9$ & $2.1 \pm 1.5$ & 0.542 \\
DAS28-CRP & $3.6 \pm 1.0$ & $2.3 \pm 0.9$ & $<0.001$ \\
SDAI & $14.0 \pm 6.9$ & $5.7 \pm 5.0$ & $<0.001$ \\
Total GSUS score & $16.9 \pm 12.6$ & $14.8 \pm 12.7$ & 0.572 \\
Total PDUS score & $11.3 \pm 9.07$ & $6.5 \pm 6.0$ & 0.076 \\
Maximum PDUS grade & $2.0 \pm 1.0$ & $1.6 \pm 0.8$ & 0.207 \\
\hline
\end{tabular}

Conclusions: Even patients have high disease activity, golimumab 100mg suppress the synovitis and tenosynovitis very well. In the condition where disease activity was sufficiently controlled, there was no difference in the synovitis findings of ultrasound at the dose of golimumab.

Acknowledgements: We wish to thank Setsuko Takeda, Ayumi Hashimoto, Emi Yamashita, Rika Morinaka, Hatsue Ueda and Tomomi Iwahashi for their special efforts as a sonographer and collecting data.

Disclosure of Interest: None declared

DOI: 10.1136/annrheumdis-2017-eular.1451

\section{AB1062 PATTERNS OF MUSCULOSKELETAL SYSTEM INVOLVEMENT IN PATIENTS WITH TYPE I AND TYPE II DIABETES MELLITUS}

T.I. Adrosy, M.I. Hanafy, H.S. Hamoud, A.A. Negm. Rheumatology Department, Faculty of Medicine, Al-Azhar Univerist, Cairo, Egypt

Background: Diabetes mellitus (DM) is a chronic disease, no known cure except in very specific situations. Musculoskeletal Utrasonography (MSUS) has a great sensitivity that can help clinical examination for the detection of peripheral enthesitis associated with DM

Objectives: To study the different patterns of musculoskeletal (MSK) system affection in both types of diabetes mellitus (DM).

Methods: We performed a retrospective single-center study on sixty five patients during the period from May 2014 to February 2015, to evaluate MSK manifestations in diabetic patients at Sayyed Galal University Hospital, Cairo, Egypt. Patients were identified as diabetics based on Diagnosis and Classification of Diabetes Mellitus diagnostic criteria (1997) (1). Clinical data, laboratory investigations, X-ray, musculoskeletal ultrasonography (MSUS) (2) and Bone mineral density was measured using Dual energy X-ray absorptiometry (DEXA) scan (3) were all collected from all patients.

Results: We included 65 diabetic patients; of these 21 patients (32.31\%) had type I diabetes while 44 patients $(67.69 \%)$ had type II diabetes. Age in type I was $24.5 \pm 10.5$ years while in type II was $50.1 \pm 8.44$ years $(P=0.001)$. DM type II showed higher BMI $(P=0.001)$, fatigue $(P=0.005)$, shoulder periarthritis (frozen shoulder) $(P=0.034)$, knee osteoarthritis $(P=0.002)$, cheiroarthropathy $(P=0.016)$, anserine bursitis $(P=0.001)$ and plantar fasciitis $(P=0.003)$ than type I. Osteoporosis was found in both types but type II showed more prevalence $13 / 44$ patients $(29.5 \%)$ while type I showed only $3 / 21(14.2 \%)$. No statistically significant difference between both groups as regard t-score in the three sites. MSUS showed increased prevalence of quadriceps tendon enthesophytes in type I $(P=0.033)$, while Infrapatellar $(P=0.023)$ and retrocalcaneal bursitis $(P=0.001)$ were more prevalent in type II DM.

Conclusions: Early evaluation of any diabetic patient regarding BMD by DEXA scan and soft tissue by MSUS seems to be beneficial for early detection of any abnormality and therefore early management and prevention of complications. References:

[1] American Diabetes Association. Diagnosis and classification of diabetes mellitus. Diabetes Care. 2013; 36

[2] Blake GM and Fogelman I. DEXA scanning and its interpretation in osteoporosis. Hosp Med 2003; 64:521-5.

[3] Balint PV, Kane D, Wilson H, Mclnnes IB and Sturrock RD: Ultrasonography of entheseal insertions in the lower limb in spondyloarthropathy. Ann Rheum Dis 2002 ; 61:905-910.

Disclosure of Interest: None declared

DOI: 10.1136/annrheumdis-2017-eular.2420

\section{AB1063 ULTRASONOGRAPHIC ASSESSMENT OF SYNOVITIS IN PATIENTS WITH LESSOR TOE DEFORMITY DUE TO RHEUMATOID ARTHRITIS}

T. Kashiwagura ${ }^{1}$, Y. Kimura ${ }^{2}$, I. Wakabayashi ${ }^{3}$, M. Fujii ${ }^{2}$, M. Kobayashi ${ }^{4}$, M. Urayama ${ }^{5}$, T. Aizawa ${ }^{6}$, Y. Sugimura ${ }^{7}$, H. Aonuma ${ }^{8}$, N. Miyakoshi ${ }^{9}$, Y. Shimada ${ }^{10} .{ }^{1}$ Department of Orthopedic Surgery, Akita City Hospital; ${ }^{2}$ Department of Orthopedic Surgery; ${ }^{3}$ Department of Rehabilitation Medicine, Akita City Hospital, Akita; ${ }^{4}$ Department of Orthopedic Surgery, Hiraka General Hospital, Yokote; ${ }^{5}$ Department of Orthopedic Surgery, Ogachi General Hospital, Yuzawa; ${ }^{6}$ Department of Orthopedic Surgery, Kita Akita Municipal Hospital, Kita Akita; ${ }^{7}$ Department of Orthopedic Surgery, Nakadori general hospital, Akita; ${ }^{8}$ Kakunodate general hospital, Senboku; ${ }^{9}$ Akita University Graduate school of Medicine, Akita, Japan; ${ }^{10}$ Department of Orthopedic Surgery, Akita University Graduate school of Medicine, Akita, Japan

Background: In recent years, joint ultrasonography has been widely used for the diagnosis and treatment of rheumatoid arthritis (RA), allowing visualization of synovitis. Its clinical usefulness in early diagnosis and evaluation of disease activity has been reported. Continuous inflammation, osteochondral destruction, and soft tissue destruction due to synovitis in toe joints result in various clinical pictures of the foot. In the lateral toes in the forefoot, subluxation or luxation of the metatarsophalangeal (MTP) joints may occur, leading to painful callosities and resultant disturbance in activities of daily living. Few reports have addressed toe deformity and joint ultrasonographic findings of synovitis in the forefoot.

Objectives: In this study, lateral MTP joints were assessed using joint ultrasonography in RA patients to examine the correlation with deformity.

Methods: Seventy feet of 61 RA patients were examined in the outpatient clinic of our hospital. Patients who underwent surgery were excluded. The mean age of the patients was 66 years (24 to 92 years), and the mean duration of disease was 12 years and 9 months ( 1 month to 40 years). Biologic products were used for 23 feet. Joint ultrasonography was performed by the same examiner, using the same room and apparatus. Synovitis was defined as Grade 1 or more as determined by the power Doppler method. Based on foot radiographs in upright position obtained before and after ultrasonography, patients with luxation, subluxation, and joint fissure narrowing were classified into the deformity group, those with bone erosion and geode formation into the bone erosion group, and lack of abnormal findings into the normal group. 